\title{
Effect of miR-204\&211 and RUNX2 control on the fate of human mesenchymal stromal cells
}

Benedetto Sacchetti ${ }^{1}$, Alessandro Fatica ${ }^{3}$, Melissa Sorci ${ }^{3}$, Antonio Sorrentino ${ }^{4}$, Michele Signore ${ }^{2}$, Annamaria Cerio ${ }^{2}$, Federica Felicetti ${ }^{2}$, Alessandra De Feo ${ }^{2}$, Elvira Pelosi ${ }^{2}$, Alessandra Caré ${ }^{2}$, Edoardo Pescarmona ${ }^{5}$, Jan Oxholm Gordeladze $^{6}$, and Mauro Valtieri ${ }^{2,7, *}$

${ }^{1}$ Department of Science, University ROMA TRE, Rome, Italy

2 Department of Oncology and Molecular Medicine, Istituto Superiore di Sanità, Rome, Italy

${ }^{3}$ Department of Biology and Biotechnology Charles Darwin, "La Sapienza" University, Rome, Italy

${ }^{4}$ Exiqon A/S, Skelstedet 16, 2950 Vedbæk, Denmar

5 "Regina Elena" National Cancer Institute, Rome, Italy

${ }^{6}$ Institute of basic Medical Science, Department for Molecular Medicine, Section for Biochemistry, University of Oslo, Oslo, Norway

${ }^{7}$ Sbarro Institute for Cancer Research and Molecular Medicine \& Center of Biotechnology, College of Science and Technology, Temple University, Philadelphia, Pennsylvania

Received 21 September 2017, Accepted 9 November 2017, Published online 5 December 2017

\begin{abstract}
MiR-204 and 211 enforced expression in murine mesenchymal stromal cells (MSCs) has been shown to induce adipogenesis and impair osteogenesis, through RUNX2 down-modulation. This mechanism has been suggested to play a role in osteoporosis associated with obesity. However, two further fundamental MSC functions, chondrogenesis and hematopoietic supporting activity, have not yet been explored. To this end, we transduced, by a lenti-viral vector, miR-204 and 211 in a model primary human MSC line, opportunely chosen among our MSC collection for displaying all properties of canonical bone marrow MSCs, except adipogenesis. Enforced expression of miR-204\&211 in these cells, rescued adipogenesis, and inhibited osteogenesis, as previously reported in murine MSCs, but, surprisingly, also damaged cartilage formation and hematopoietic supporting activity, which were never explored before. RUNX2 has been previously indicated as the target of miR-204\&211, whose down modulation is responsible for the switch from osteogenesis to adipogenesis. However, the additional disruption of chondrogenesis and hematopoietic supporting activity, which we report here, might depend on diverse miR-204\&211 targets. To investigate this hypothesis, permanent RUNX2 knock-down was performed. ShRUNX2 fully reproduced the phenotypes induced by miR-204\&211, confirming that RUNX2 down modulation is the major event leading to the reported functional modification on our MSCs. It seems thus apparent that RUNX2, a recognized master gene for osteogenesis, might rule all four MSC commitment and differentiation processes. Hence, the formerly reported role of miR204\&211 and RUNX2 in osteoporosis and obesity, coupled with our novel observation showing inhibition of cartilage differentiation and hematopoietic support, strikingly resemble the clinical traits of metabolic syndrome, where osteoarthritis, osteoporosis, anaemia and obesity occur together. Our observations, corroborating and extending previous observations, suggest that miR-204\&211-RUNX2 axis in human MSCs is possibly involved in the pathogenesis of this rapidly growing disease in industrialized countries, for possible therapeutic intervention to regenerate former homeostasis.
\end{abstract}

Keywords: Mesenchymal stromal cells, miR-204\&, RUNX2, cartilage, hematopoietic support activity, osteogenesis, adipogenesis

\section{Introduction}

Mesenchymal stromal cells (MSCs) represent the second stem cell population residing in bone marrow (BM) [33,37], wherein they provide both the reservoir for

\footnotetext{
* mauro.valtieri@iss.it
}

skeletal cell turn-over and "the soil" for parenchymal cells "seeds", i.e. hematopoietic stem cells (HSCs). MSCs are endowed with extensive proliferation, equal or longer than three months in vitro, the ability to differentiate in at least four structural lineages, osteoblasts, chondroblasts, adipoblasts and reticular stromal cells supporting HSCs; the capability to produce huge amounts of multiple growth 
factors $[33,37]$ underlying their anti-apoptotic, immuneregulator, nurturing and regenerative potential [30,33,37]. Although MSCs were first discovered in BM, other tissuespecific MSCs have been recently identified and categorized on the basis of their antigen expression signatures and cell differentiation spectra. Tissue-specific MSCs partly overlap with pericytes and span aubiquitous distribution in human tissues as structural cells [24,30]. We first isolated and characterized, through four lineages, MSCs in bone marrow [33]. Subsequently, we isolated and banked tissuespecific MSCs from amnion, foetal liver, tonsil, dental pulp, lung, bowel $[24,30]$ and dermal connective tissue. Colonic (C-) MSCs were subjected to in depth analysis and comparison to BM-MSCs, classified, both functionally and by smooth muscle actin expression, in peri-cryptic and inter-cryptic myofibroblasts [24,30]. Apical peri-cryptic myofibroblasts are in close contact with intestinal epithelial stem cells, wherein they nurture, regulate and maintain them in the intestinal niche, similarly to the hematopoietic niche in the BM. Apical or peri-cryptal myofibroblasts hierarchically represent the highest intestinal MSC rank, i.e. the closest to BM-MSCs. Inter-cryptal MSCs, on the other hand, represent the progeny of the former, mainly devoted to the structural function of holding the crypts in place. In the present study we utilized primary peri-cryptic C-MSCs endowed with all the BM-MSCs functions except adipogenesis, to assay the effect of enforced expression of miR-204\&211 on their whole differentiation spectrum.

MicroRNAs (miRNAs) are 20-22 nt long single stranded RNAs that regulate gene expression by targeting complementary nucleic acid sequences $[7,28,32,1,22,34,13,3]$. Micro-RNAs play a regulatory role in virtually all cellular functions, including stemness [32], plasticity [1] and differentiation in many stem cell types including MSCs [32,1,22,34,13,3,11]. A growing clinical interest is arousing around miRas diagnostic and therapeutic tool for several diseases. MiR-204 and 211 enforced expression in murine MSCs have been previously shown to induce adipogenesis and repress osteogenesis, by targeting RUNX2 [14,17]. Being bone formation inter-wined with chondrogenesis, we seek to explore their effect of on cartilage formation. In bone development, in fact, chondro- and osteo-genesis are coordinated developmental processes, since bones form from cartilage cores by endochondral ossification $[8,18,5]$ head and neck bones excluded for carrying out membranous ossification. An overlooked but pivotal lineage, functionally defined as hematopoietic supporting activity, is also dependent on MSCs, as constituents of the so called "hematopoietic niche", i.e. the soil for HSCs and their siblings [29]. The "hematopoietic niche" is a micro-structural entity whose best candidate components are reticular stromal cells and osteoblasts, both pertaining to the MSC lineage $[26,31,35,29]$. Prototypes of the "hematopoietic niche" are the blood islands around the developing embryo or the erythroblast islands in the red BM.

However, the effect of miR-204\&211 on both chondrogenesis and hematopoietic supporting activity has been thus far neglected. One major target of miR-204\&211 is
RUNX2, a known master gene for bone development [4]. We report here that miR-204\&211, formerly described to inhibit osteogenesis and promote adipogenesis [14] in murine MSCs, reproduce this phenothypein human CMSCs and additionally inhibit chondrogenesis and hematopoietic supporting activity.

Noteworthy, further targets of miR-204\&211, besides RUNX2, might account for the disruption of chondrogenesis and hematopoietic supporting activity we report.

Regarding chondrogenesis, we explored CRTAP [5] a reported virtual target according to Target Scan6.2 (www. targetscan.org) but it was not decreased by miR-204\&211. We then performed RUNX2 knock-down and fully reproduced the miR-204\&211 over-expression phenotype, demonstrating that RUNX2 itself controls all four canonical MSC differentiation lineages, including cartilage formation and hematopoietic supporting activity.

\section{Materials and methods}

\subsection{Isolation and characterization of MSCs}

The isolation procedure from human colon biopsies was derived from the BM-MSC isolation [33] detailed below. BM from healthy donors was purchased from CAMBREX Poietics cell systems (Gaithersburg, MD). BM samples were treated for $20 \mathrm{~min}$ at $20^{\circ} \mathrm{C}$ with RosetteSep human MSC enrichment cocktail (StemCell Technologies, Vancouver, BC, Canada) composed by CD3, CD14, CD19, CD38, CD66b, Glycophorin A tetrameric antibody $(\mathrm{Ab})$ complexes crosslinking unwanted cells with red blood cells, diluted, and centrifuged over Ficoll-Hypaque gradient for $25 \mathrm{~min}$ at $300 \mathrm{~g}$ at $20^{\circ} \mathrm{C}$. Enriched cells were collected, washed, and treated with $\mathrm{NH}_{4} \mathrm{Cl}$ (StemCell) for $10 \mathrm{~min}$ in ice to remove residual red blood cells. CD34 cells were removed by MACS column (Miltenyi, Bergisch Gladbach, Germany). Enriched cells were then cultured at sub-clonal density $\left(1-10\right.$ cells $\left./ \mathrm{cm}^{2}\right)$ for 3 weeks in a-medium (Invitrogen, Carlsbad, CA), 20\% fetal calf serum (FCS; StemCell) in T75 flasks at $37^{\circ} \mathrm{C}$ in $5 \% \mathrm{CO}_{2} / \mathrm{O}_{2}$ atmosphere. Half medium was replaced two times a week until MSCs reached confluence, defined as passage (P) 0. MSCs were detached by trypsin-ethylenediaminetetraacetic acid solution (Invitrogen) treatment, partly freezed and partly replated. Each weekly replating or $\mathrm{P}$ was defined by a progressive number. Colon surgical specimens, obtained from patients who signed an informed consent before undergoing surgical resection of a tumor lesion, were dissected to separate the compromised from the normal tissue, the normal tissue fragment was thoroughly washed with PBS supplemented with 5X antibiotic/antimycotic (A/A) solution (Invitrogen), maintained $\mathrm{o} / \mathrm{n}$ in $\mathrm{PBS} 5 \mathrm{X} \mathrm{A} / \mathrm{A}$ at $4{ }^{\circ} \mathrm{C}$, treated with 30-45 $\mathrm{ml} 1 \mathrm{mM}$ EDTA/EGTA PBS $75^{\prime}$ at $20^{\circ} \mathrm{C}$, vigorously shaked, then processed as described for BM.

Cells are cultured in controlled $\mathrm{CO}_{2} / \mathrm{O}_{2}$ environment provided by Forma Scientific incubators customized by BioSpherix C-Chambers and ProOx110 controller for normoxia or hypoxia settings. 


\subsection{Isolation of cord blood (CB) CD34 ${ }^{+} \mathrm{HPCs}$}

Collection of $\mathrm{CB}$, isolation of $\mathrm{CD}^{+} 4^{+}$cells were performed as described $[36,39]$.

\subsection{Hematopoietic support}

BM-MSCs and colon (C)-MSCs were plated at the saturating density for confluence of $3.6 \times 10^{4} / \mathrm{cm}^{2}$ in 96 well/plates (Falcon, Franklin Lakes, NJ) in alphamedium, 20\% MCS selected FCS (Lonza); 1 week before the start of the long-term culture (LTC) assay [36,39] the medium was changed to alpha-medium with $10 \%$ FCS, 10\% Horse S (both from Stem Cell Technologies), supplemented with $10^{-6} \mathrm{M}$ hydrocortisone (Sigma, St Louis, MO) [36,39]. Plates were then seeded with $2 \times 10^{3}$ $\mathrm{CD} 4^{+}$cord blood (CB)cells and cultured up to 5 weeks at $33{ }^{\circ} \mathrm{C}$ in $5 \% \mathrm{CO}_{2} / \mathrm{O}_{2}$ atmosphere [36,39]. Half volume of each culture $(0.03 \mathrm{ml})$ was harvested weekly and replaced with fresh medium. Cells weekly recovered from the harvested medium were counted and seeded in hematopoietic multilineage semi-solid clonogenetic assay cultures for 14 days at $37^{\circ} \mathrm{C}$ in $5 \% \mathrm{CO}_{2} / \mathrm{O}_{2}$ atmosphere [36,39] in order to allow the growth and differentiation of hematopoietic colonies. At week 5, upon termination of LTC cultures, both cells recovered from the medium and the cells entrapped in the MSC layers were independently counted and seeded in hematopoietic clonogenetic assays $[36,39]$. Hematopoietic colonies were scored as CFUGEMM (Colony Forming Unit-Granulocyte Erythroid Megacaryocyte Monocyte: large mixed red and white colonies) BFU-E (Burst Forming Unit-Erythroid: pure erythroid red colonies) and CFU-GM (white granulocyte and/or monocyte colonies) after 2 further week secondary culture under an Olympus inverted microscope.

\subsection{MSCs proliferation}

BM- and C-derived MSCs were cultured in $75 \mathrm{~cm}^{2}$ flasks, re-fed twice a week with $12.5 \mathrm{ml}$ of fresh medium, detached at confluence, usually once a week, by trypsinethylenediaminetetraacetic acid solution (Invitrogen) treatment, counted, partly frozen and partly reseeded for culture at $37^{\circ} \mathrm{C}$ in $5 \% \mathrm{CO}_{2} / \mathrm{O}_{2}$ atmosphere. Each weekly re-seeding or passage $(\mathrm{P})$ was defined by a progressive number.

\subsection{In vitro MSC differentiation}

Osteogenic. $3.1 \times 10^{3} \mathrm{MSC} / \mathrm{cm}^{2}$ were seeded in sixwell/plates(BD) in MSCGM (Cambrex, Poietics Cell Systems) for $24 \mathrm{~h}$ at $37^{\circ} \mathrm{C}$ in $5 \% \mathrm{CO}_{2} / \mathrm{O}_{2}$ atmosphere. MSCGM was replaced with Osteogenesis Induction Medium (Cambrex), and MSCs cultured for 3 weeks with refeeding every 3-4 days. Osteoblasts were stained with ALP and Von Kossa, labeling early and late differentiation, respectively. Alternatively, in the RUNX2 knock-down, osteogenic differentiation was assessed by Von Kossa's stain alone.

Chondrogenic. $2.5 \times 10^{5} \mathrm{MSC}$ were washed twice at $150 \mathrm{~g}$ for $5 \mathrm{~min}$ at $20^{\circ} \mathrm{C}$ with incomplete chondrogenic medium (Cambrex), re-suspended in $0.5 \mathrm{ml}$ complete medium (Cambrex) in $15 \mathrm{ml}$ polypropylene tube and cultured for 3 weeks at $37^{\circ} \mathrm{C}$ in $5 \% \mathrm{CO}_{2} / \mathrm{O}_{2}$ atmosphere, re-feeding every $2-3$ days with fresh medium. In RUNX2 knock-down experiments, for immunohistochemistry and immuno-localization studies, cartilage pellets were fixed in $4 \%$ formaldehyde in phosphate buffer, processed for paraffin embedding and sectioned serially. Five-micronthick sections were stained with hematoxylin and eosin (Sigma-Aldrich) or Alcian Blue (Sigma-Aldrich). Immuno-histology experiments were performed using primary antibodies anti-human type II collagen (Col II; ab53047, Abcam), anti-human type X collagen (Col X; ab49945, Abcam) and anti-human Sox-9 (sc-20095, Santa Cruz Biotechnology). Immuno-localization was completed with the use of standard peroxidase/diaminobenzidine (DAB) reaction and counterstained with hematoxylin. Bright-field light microscopy images were obtained with the use of a Zeiss Axiophot epifluorescence microscope (Carl Zeiss, Oberkochen, Germany).

Adipogenic. Confluent MSCs were supplemented with Adipogenesis induction medium for 3 days followed by 1-3 days of culture in maintenance medium (both from Cambrex). After three cycles of induction/maintenance, cells were cultured for an additional week with maintenance medium at $37^{\circ} \mathrm{C}$ in $5 \% \mathrm{CO}_{2} / \mathrm{O}_{2}$ atmosphere. Adipocytes maturation was checked in phase contrast microscopy, then cultures were fixed and intracellular lipid droplets stained with oil red-O solution (SigmaAldrich), for further microscope analysis.

\subsection{Real time qRT-PCR}

RNA was isolated from cells using the "Total RNA Purification micro Kit" (NorgenBioteK Corp, Canada) according to the manufacturer's protocol. Real time quantification was performed according to the TaqMan technology (Applied Biosystems, Foster City, CA, USA): miR-204 (hsa-miR-204-5p) \#000508; miR-211 hsa-miR211-5p \#000514; RNU6B (\#001093).

\subsection{Lenti-miR204/211 plasmids generation}

The Lenti-miR204 and Lenti-miR211 plasmids were generated by cloning a fragment of the pri- miRNA (from -100 to +135 bp relative to the $5^{\prime}$-end of mature miR-204 and from -103 to +143 relative to the $5^{\prime}$-end of mature miR-211, respectively) into the pSP65-U1 cassette plasmid [7]. The expression cassette was then subcloned into the the EcoRV site of the lentiviral vector pRRLcPPT.hPGK.EGFP.WPRE [7]. Empty vetor was utilized as control (PGK). Infective particles were produced and utilized as previously described $[7,20]$.

\subsection{RUNX2 knockdown}

RUNX2 knockdown was obtained by Mission Lentiviral shRNA clone SHCLNV-NM 004348 targeting RUNX2 (Sigma-Aldrich, USA). Mission Lentiviral Non-Targeting shRNA clone SHC002 (Sigma-Aldrich, USA) was utilized as control. Lentiviral particles were 
prepared according to the manufacturer's specifications. Infection of C3 MSCs was performed as previously described [28].

\subsection{Western blot analysis}

Western blot analysis was performed according to standard procedures either on cultured cells or on chondrogenic spheres. Antibodies listed below were used in accordance to the manufacturer's instructions: RUNX2 (27-K: sc-101145) (Santa Cruz Biotechnology Dallas, TX, USA); $\beta$-ACTIN (Oncogene Research, La Jolla, CA, USA) and GAPDH (2D9) (OriGene, Rockville, MD, USA). $\beta$-ACTIN and GAPDH were used as loading controls.

\subsection{Statistical analysis}

Statistical analysis was performed by one-way ANOVA and subsequently by Bonferroni post-tests. Differences are considered statistically significant at $p<0.05$.

\section{Results}

\subsection{MicroRNA $204 \& 211$ inhibit bone and restore fat differentiation, but inhibit also cartilage and hematopoietic support in human MSCs}

Intrigued by a former article describing the role of miR204\&211 in murine MSC differentiation [14], we decided to reproduce these data on our primary human MSCs. To this end, among the best characterizes MSC lines in our collection [30], we chose the human smooth muscle actin $(\mathrm{SMA}+)$ peri-cryptic colonic C3-MSCs, comparable, for their differentiation profile, to the murine cells originally studied in [14]. The differentiation profile of C3-MSCs is shown in Figure 1. Human wild-type C3 MSCs, and control PGKC3 MSCs differentiate along the osteogenic (Fig. 1A,a) and chondrogenic pathways (Fig. 1A,c), but not toward the adipogenic one in specific differentiation culture systems (Fig.1A,b). C3 MSCs over-expressing miR-204 or miR-211 partially gained the ability to produce adipocytes (Fig. 1A,b) and decreased the attitude to differentiate in osteocytes (Fig. 1A,a), as expected [14]. As a novel finding, since chondrogenesis was not explored before, miR204 and miR211 impaired cartilage development (Fig. 1A,c). We formerly showed that wild-type C3MSCs could support hematopoiesis in a $5 \mathrm{wk}$ long-term culture (LTC) system as efficiently as BM MSCs [30]. We, hence, decided to evaluate this function in C3-MSCs overexpressing miR-204 or miR-211 in LTC to measure their support for cord blood CD 34+ hematopoietic progenitors (HPCs), in vitro. In this system, C3 MSCs over-expressing miR-204 or miR-211maintained the overall hematopoietic cell number (Fig. 1B, a) similarly to parental cells, but were largely ineffective in supporting hematopoietic progenitors (Fig. 1B,b), when compared to control mock (PGK) transduced C3-MSCs, as evaluated in secondary clonogenic cultures in semisolid medium derived from the primal culture. Wild-type BM12-MSCs were included as LTC-system control, representing standard hematopoietic supportive cells.

The selectivity of progenitor cell decrease, together with the maintenance of the total cell number, counted by dye exclusion, ruled out any not-specific, toxic effect dependent from C3-MSC infected with miR-204 or 211 (Fig. 1B, a vs b).

\subsection{MicroRNA 204 \& 211 inversely correlate with RUNX2, impairing cartilage differentiation}

Human C3-MSCs, have little steady state expression of miR-204\&211 (Fig. 2 A), similarly to reference human BM BM12-MSCs (Fig. 2A) whereas SMA- inter-cryptic colonic C6-MSCs [30) express higher levels of miR-204/ 211 (Fig. 2A), being a more differentiated cell class. C12 is an additional SMA + colonic MSC line similar to C3 [30].

This observation may suggest that the low expression of miR-204\&211 is linked to a less differentiated cell state. PGK, miR-204 and 211 infected C3 MSCs were induced in cartilage spheroids for 1 week, then lysed for protein extraction (Fig. 2B). Western Blot (WB) analysis demonstrated that RUNX-2 protein, a key transcription factor for osteogenesis, was highly expressed in control PGKC3 MSCs (Fig. 2B). In contrast, C3 MSCs over-expressing miR-204 or miR-211, effectively down-modulated RUNX-2 (Fig. 2B), whereas expression levels of CRTAP gene product, a key gene for chondrogenesis [5], and a potential target for miR-204\&211, according to Target Scan6.2 (www.targetscan.org), were not altered. GAPDH, the control protein, remained steady (Fig. 2B). Additional WB analysis, in undifferentiated SMA+ C3 MSCs demonstrated that RUNX-2 was expressed as well (Fig. 2C, third lane) whereas it was not in SMA-C6 MSCs (fifth lane) nor in C13 (fourth lane), an additional SMA-colonic MSC line [30] (Fig. 2C). More importantly, it was sharply decreased in C3 over-expressing miR-204 or miR-211 (Fig. $2 \mathrm{C}$, second and first lane, respectively). Actin control protein, used here, was steady (Fig. 2C). During ex vivochondrogenesis (Fig. 2D, a) RUNX2 protein expression was maintained up to 2 weeks, then down-regulated at later times. It was again down-regulated upon miR-204 or miR-211 over-expression (Fig. 2D, b) in an additional experiment confirming the former results (in Fig. 2C).

\section{3 shRUNX2 inhibits bone and restore fat differentiation, similar to $\mathrm{miR} 204 \& 211$ overexpression}

To rule out that further miR-204\&211 targets might account for the disruption of chondrogenesis and hematopoietic supporting activity in C3 MSCs over-expressing miR-204\&211, we permanently knocked-down RUNX2 in C3 MSCs with a lentivirus containing RUNX2 shRNA infection. Also in these experiment, the adipogenic potential was restored (Fig. 3a) whereas the osteoblastic differentiation was impaired (Fig. 3b), reproducing the phenotype obtained by miR-204 or 211 over-expression in Fig. 1A, (b) and (a). 
A

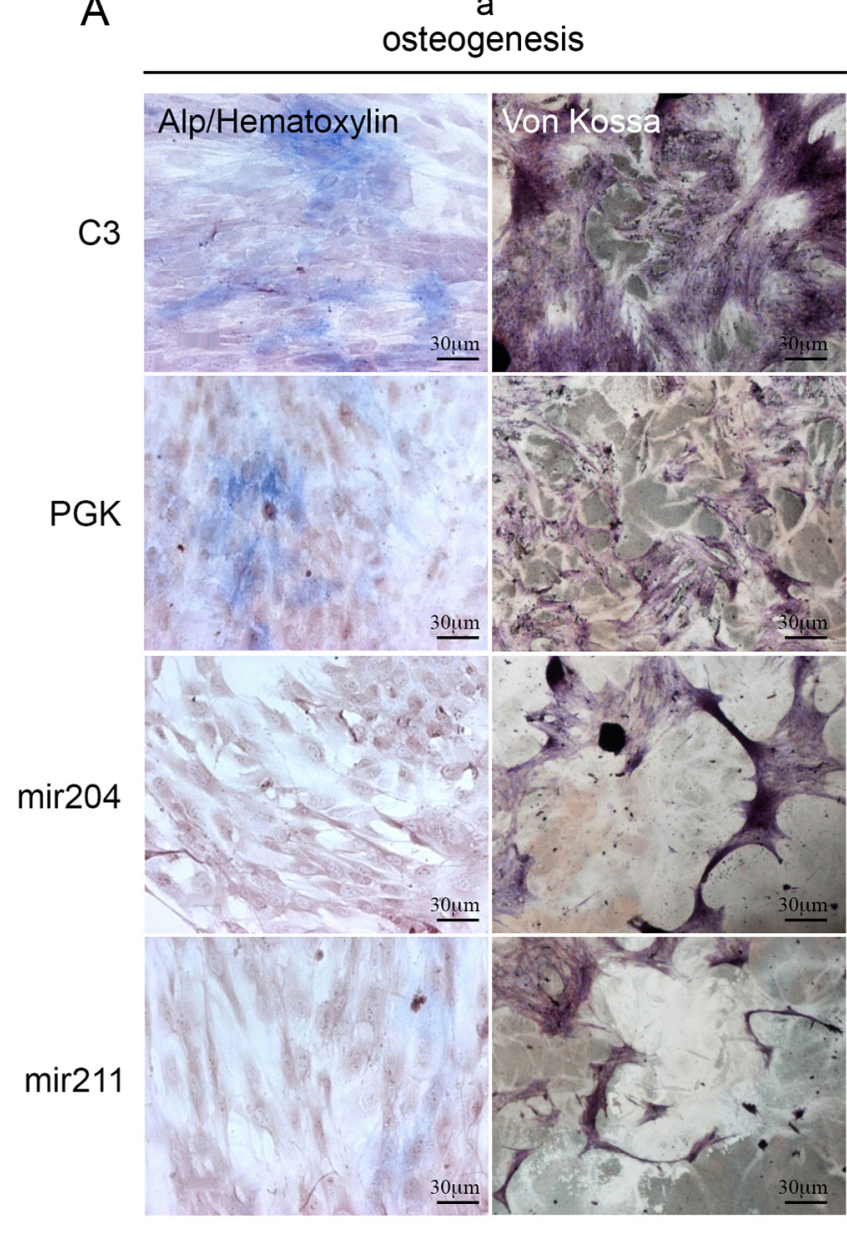

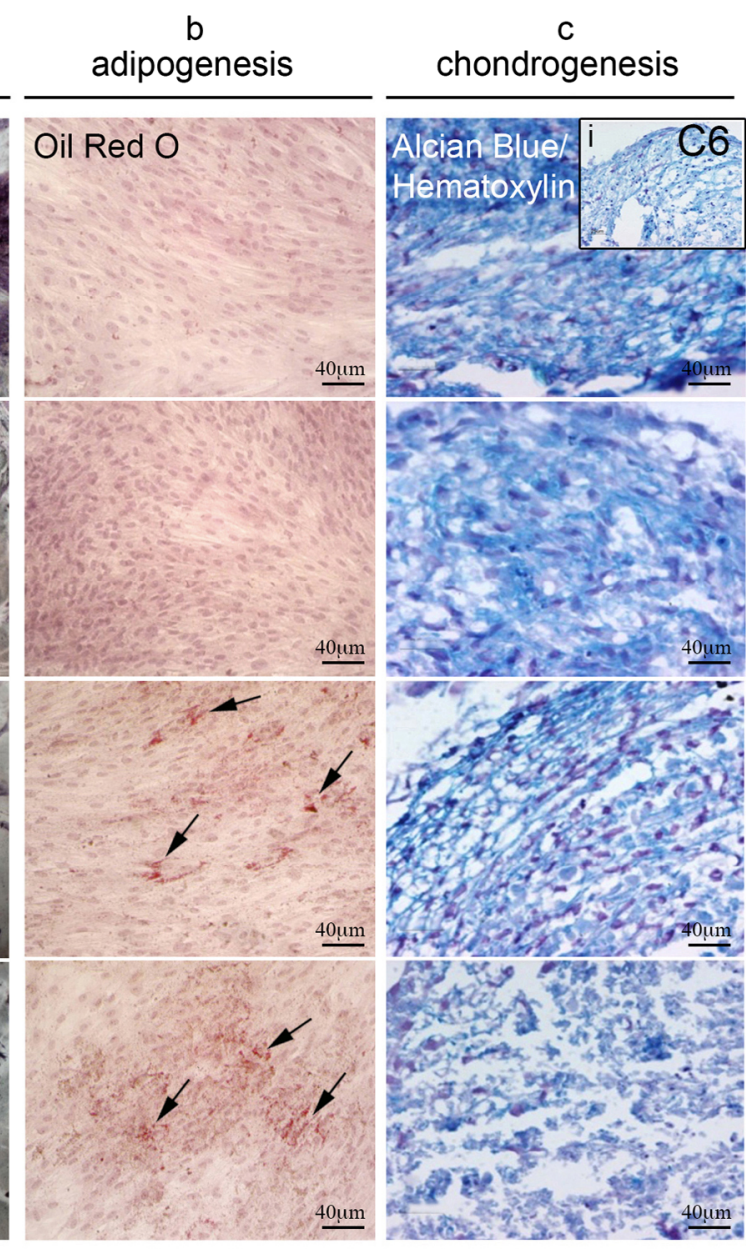

b
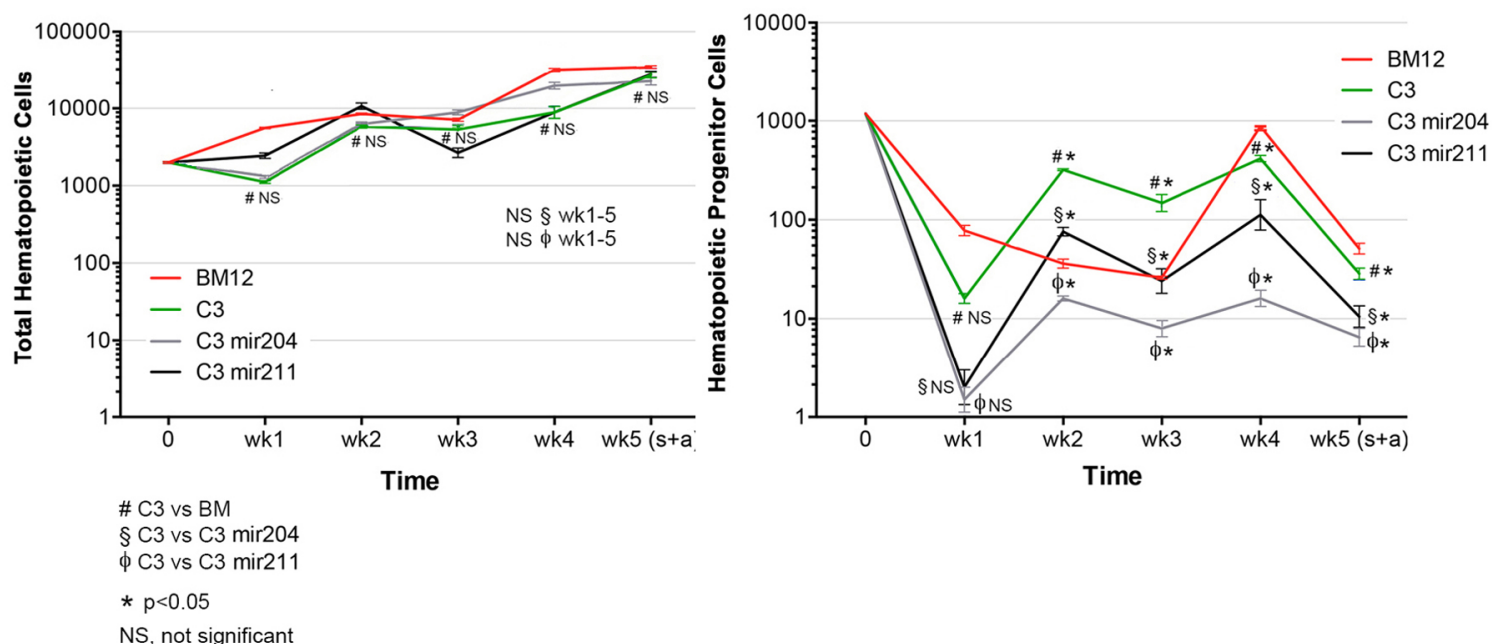

Fig. 1. (A) MiR-204 and miR-211 inhibition of osteogenesis (a), and chondrogenesis (c) coupled with promotion of adipogenesis (b) in human C3 colonic MSC line (as indicated by black arrows for intracellular lipid droplets formation). PGK represent C3 MSCs infected with empty vector viral particles. Human C6 colonic MSC line (insert, i) is shown as negative control being unable to differentiate in cartilage [30]. Scale bar $=30 \mu \mathrm{m}, 4-0 \mu \mathrm{m}$. (B) Effect of miR-204 and miR-211 enforced expression in human C3 colonic MSCs hematopoietic supporting activity. Cord blood CD34+ cells were plated onto C3-MSC layers. Weekly cultures were halved to be refed, harvested cells were counted (shown in a) and replated in semisolid hematopoietic secondary cultures to evaluate their progenitor cell content (b). Graphs derive fromone of three different experiments performed in triplicates. C3 represent C3 MSCs infected with empty vector viral particles. BM12-MSCs are included as standard hematopoietic supportive cells. The total number of hematopoietic cells in LTC was unaffected by miR204\&211 (a), whereas the number of hematopoietic progenitors (HPCs) was inhibited (b). At wk 5 the culture was terminated harvesting both the cells in suspension (s) and the ones entrapped in the adherent layer (a). Their numbers were added up to generate wh $5(\mathrm{~s}+\mathrm{a})$ points. 

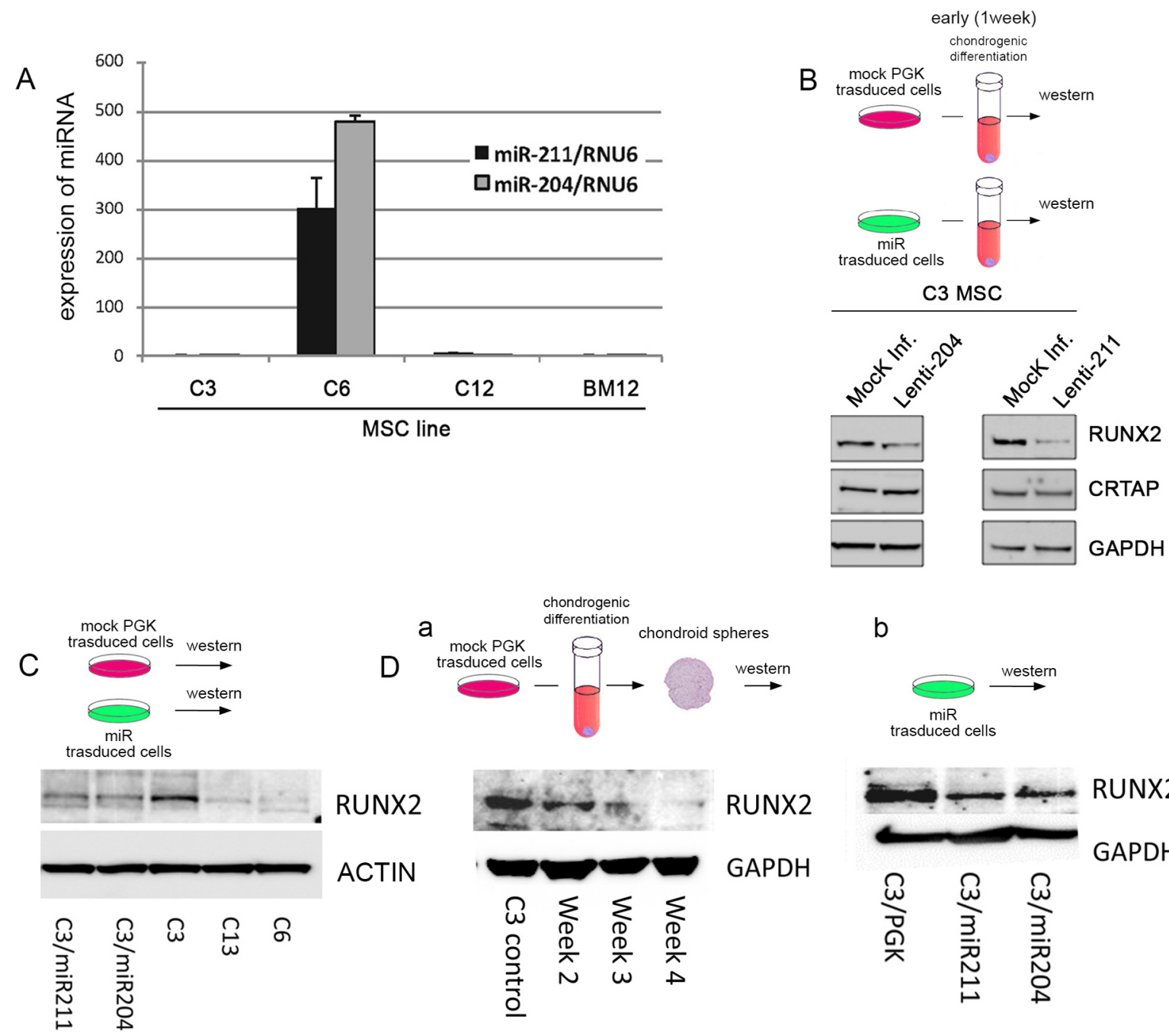

b
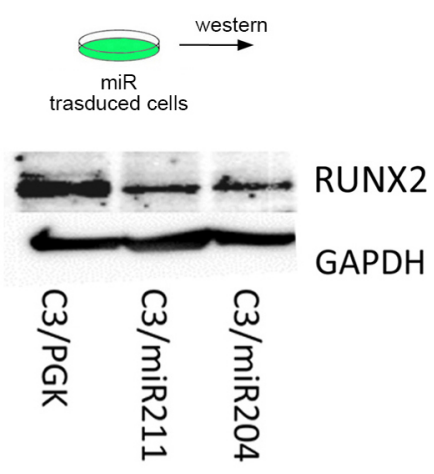

GAPDH

Fig. 2. (A) Baseline expression of miR-204 and miR-211 in undifferentiated C3, C6, C12, BM12 MSCs by Q Real Time PCR. Full scale bar graph. (B) Modulation of RUNX-2 expression. miR-204 and miR-211 overexpression inhibit RUNX-2 as evaluated by Western blot in C3 MSCs 1wk chondroid cultures. CRTAP, a cartilage master gene and a potential target of miR-204\&211 according to Target Scan6.2 (www.targetscan.org), but not miR and a (www.microrna.org), is not affected in miR 204 and miR 211 overexpressing C3 MSCs, whereas RUNX-2 is significantly decreased. GAPDH is the control protein and is steady. (C) RUNX-2 protein expression level evaluated in control C3, C3 miR204 and C3 miR211 compared with wild type C6 and C13 primary human colonic MSCs. Actin is used as control protein. (D) Modula4tion of RUNX-2 protein expression in wild type C3 MSCs upon induction of chondroid differentiation (a) or C3 over-expressing miR-204\&211 (b). RUNX-2 was down-regulated during ex vivo chondrogenesis (a) Analyses were conducted on cartilage pellets. MiR-204\&211 strongly down-regulated RUNX-2 protein expression in C3 MSCs (b).

\section{4 shRUNX2 inhibits cartilage and hematopietic support differentiation as well, similar to miR 204 \& 211 over-expression}

More interestingly, sRUNX2 shRNA human C3 MSCwere unable to differentiate in cartilage, both at macroscopic (Fig. 4 A, a) and at microscopic level (Fig. 4A, b), whereas control C3-MSCs fully differentiated to express Collagen II, X, and Sox 9 (Fig. 4A, c). Finally, sRUNX2 shRNA human C3 MSC were unable to support hematopoiesis in LTCs (Fig. 4B). The effect of shRUNX2 on C3 MSCs supporting CB CD34+ cells did not change the maintenance of the total number of cells up to 5 week in LTC (Fig. 4B, a). However, the progenitor cell fraction, within the total cell number of output in LTC, was deeply decreased when compared to control C3 MSCs or standard BM6 MSCs supportive activity (Fig. 4B, b). Thus, RUNX2 knock-down is, by itself, sufficient to replicate miR-204\&211 over-expression phenotype in C3MSC differentiation.

\section{Discussion}

Mesenchymal stromal cells (MSCs) represent the second stem cell population residing in BM [33,37] where they pair with HSCs. BM-MSCs are endowed with the ability to differentiate in four canonical lineages, osteoblasts, chondroblasts, adipoblasts and reticular stromal 


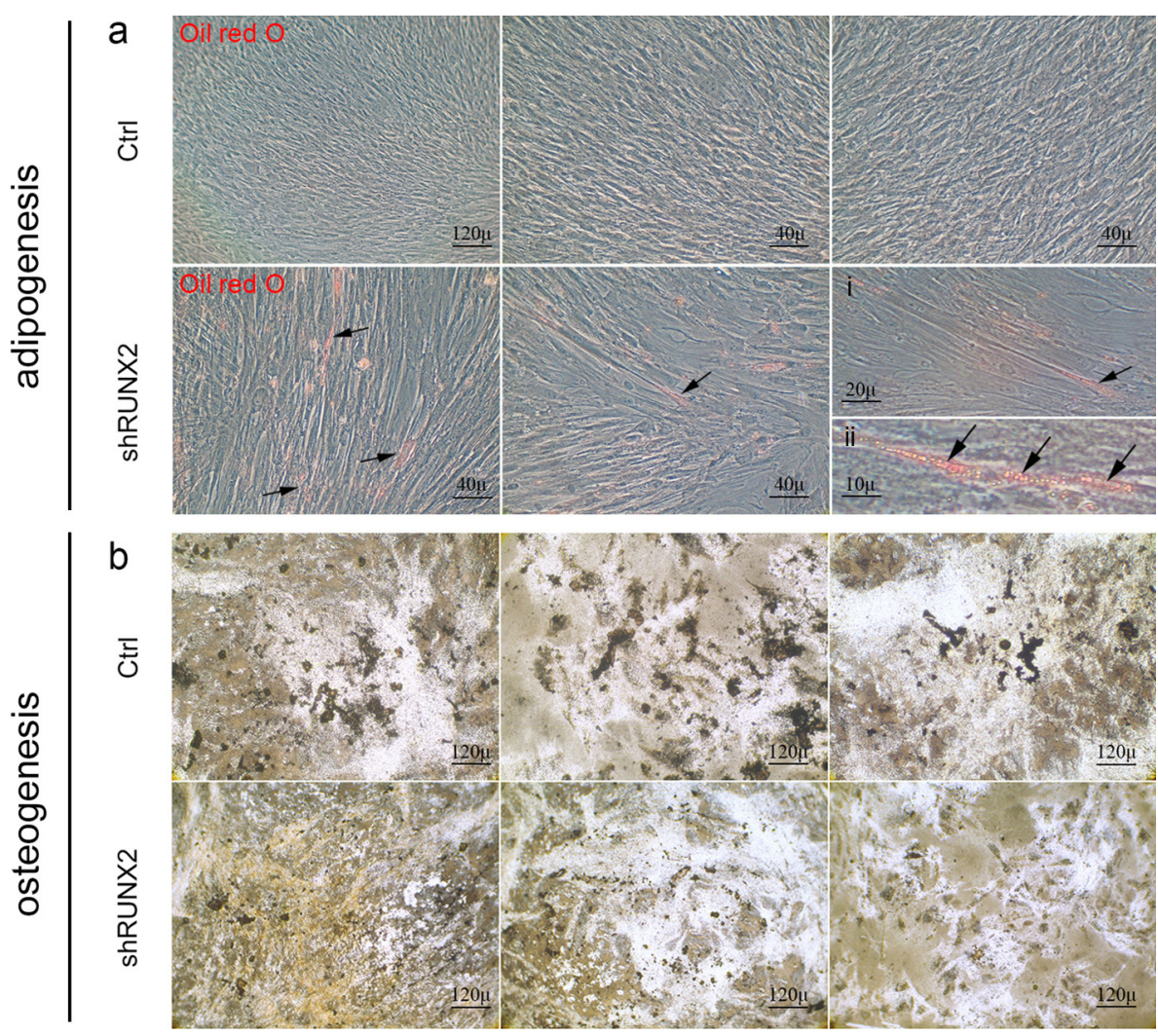

Fig. 3. Effects of RUNX2 knock-down in human C3 MSCs differentiation. (a) Oil red-O stained cultures, showing multiple areas of red lipid staining in shRUNX2 treated cells (as indicated by black arrowspointing to intracellular lipid droplets formation). (i, ii) Details of positive oil red-O-staining in treated cells, as seen in transmitted light. No droplets for adipogenic differentiation were observed in control mock treated-cells. Scale bar $=120 \mu \mathrm{m}, 40 \mu \mathrm{m}, 20 \mu \mathrm{m}, 10 \mu \mathrm{m}$. (b) Strong deposition of mineralized matrix was observed in mock control cells by Von Kossa's stain. Weak mineralization detected by Von Kossa method in shRUNX2 transduced cells. Scale bar $=120 \mu \mathrm{m}$.

cells supporting HSCs, underlying their regenerative activity. MSCs were first discovered in BM, (other) tissue-specific MSCs have been recently recognized and studied [30,27,24,2]. In this manuscript, we utilize primary peri-cryptic colonic $\mathrm{SMA}^{+} \mathrm{MSCs}$ endowed with all $\mathrm{BM}$ MSCs functions but adipogenesis [30] to explore the role of miR-204\&211 in their differentiation. Our choice was based on: (1) human C3-MSCs are endowed with most of the BM-MSCs functions except adipogenesis [30; (Fig. 1A, b)]. As apical or peri-cryptal myofibroblasts, they hierarchically represent the highest intestinal MSC rank,that is the closest to BM-MSCs. (2) Human C3MSCs, have little steady state expression of miR-204\&211 (Fig. 1B), similarly to human BM BM12-MSCs (Fig. 1B), whereas SMA- inter-cryptic colonic C6-MSCs [30] express higher levels of miR-204/211 (Fig. 1B), being a more advanced cell.

Human C6 MSCs, in fact, are unable to differentiate in cartilage (Fig. 1A, c, insert i) or to sustain hematopoiesis (not shown). In addition to $\mathrm{C} 3 \mathrm{SMA}^{+}$peri-cryptal myofibroblast line, we explored miR-204\&211 expression in an additional $\mathrm{SMA}^{+}$line, C12 [30] suggesting the low expression of miR-204\&211 as a candidate stem cell marker. Inter-cryptal SMA-MSCs, like C6 or C13 [30) represent the progeny of the $\mathrm{SMA}^{+}$peri-cryptal or apical myofibroblasts and are mainly devoted to structurally hold the intestinal crypts folded. MicroRNAs (miRNAs) are 20-22 nt not coding single stranded RNAs that fine tune gene expression by targeting complementary nucleic acid sequences $[7,28,32,1,22,34,13,3]$. Micro-RNAs have been established to play a regulatory role in stemness [32], plasticity [1] and in differentiation in many stem cell types including MSCs [32,1,22,34,13,3,11].

MiRNAs are necessary for the development of normal cartilage, as shown in mice with chondrocytes lacking the Dicer gene, a key enzyme for micro RNA maturation, with severe skeletal defects [15]. Many miRNAs have been linked to cartilage development $[38,9]$. However, to date, no report indicates a role for miR-204\&211 in chondrogenesis, despite the fact that they impair osteogenesis and by default stimulate adipogenesis, via RUNX2 inhibition [14]. RUNX2 is a recognized master gene for bone development [4]. In the developing human skeletal system, head and neck excluded, chondro- and osteo-genesis are strictly inter-wined processes. Bone formation starts from cartilage cores in a path known as endochondral ossification $[8,18,5,12]$.

The fourth canonical differentiation pathway for MSCs, often neglected, is the hematopoietic supporting activity. Although the "hematopoietic niche" is slowly 

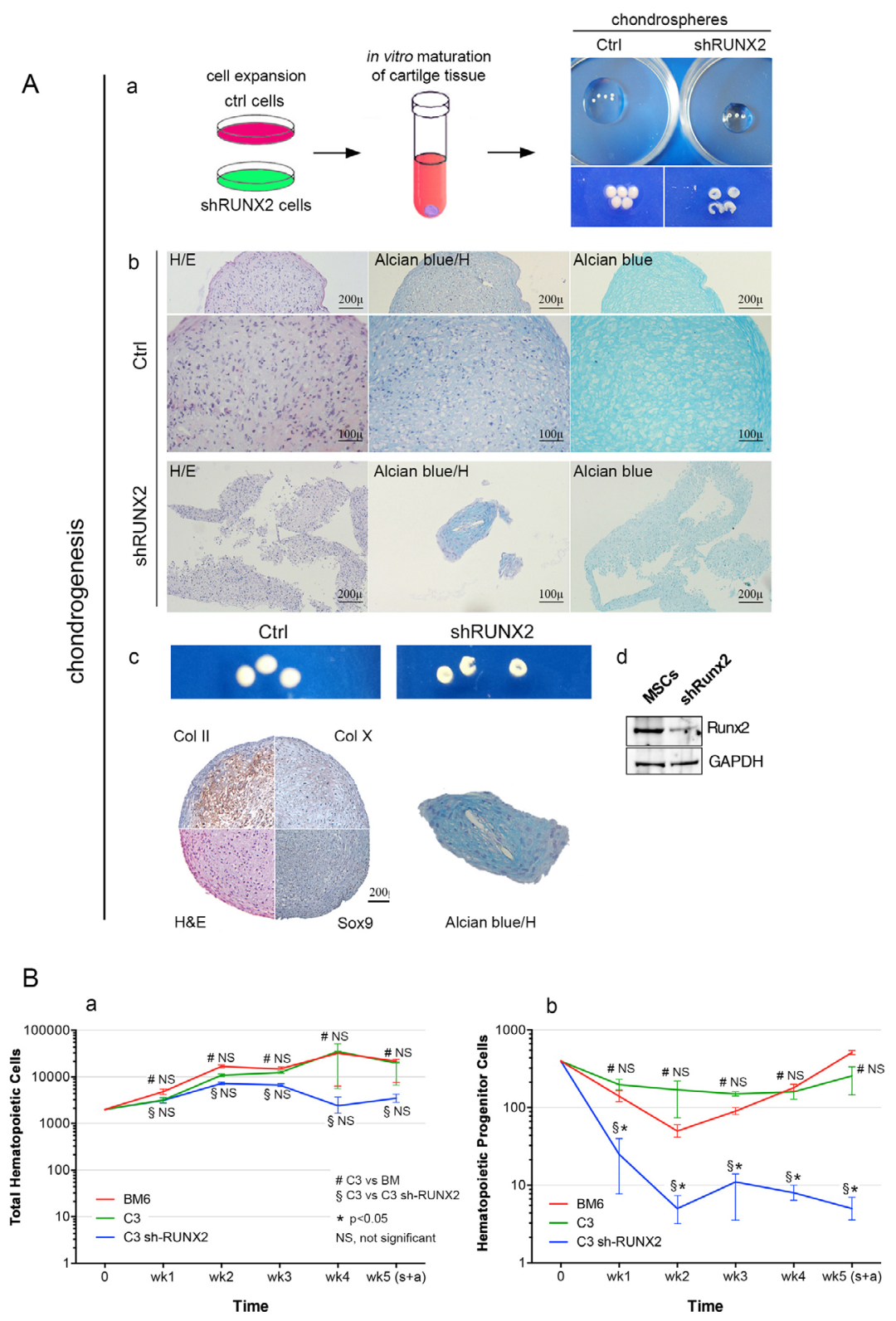

Fig. 4. Chondrogenic differentiation capability of C3-MSCs and C3 MSCs RUNX2 knock-down (Aa) Macroscopic views of 5 chondroid spheres made from treated C3 MSCs (shRUNX2), and 5 identically processed control pellets generated from mock treated C3 MSCs (ctrl). (b) Chondrospheres from mock cells generate satisfactory cartilage. In contrast, treated cells regularly failed to form good cartilage differentiation. Hematoxylin/Eosin (H/E); Alcian blue/Hematoxylin (Alcian blue/H); Alcian blue stain. Scale $\mathrm{bar}=200 \mu \mathrm{m}, 100 \mu \mathrm{m}$. (c) Macroscopic views of 3 chondroid spheres generated from C3 MSCs, and 3 pellets generated from C3 MSCs shRUNX2 are shown. Hematoxylin and eosin staining of C3-MSCs (left) pellets induced by culture with chondrogenic medium (Cambrex) for 3 weeks. Immunostaining of C3-MSCs (left) pellets with anti-human type II collagen (COL II), anti-human type X collagen (COL X) and anti-human Sox-9 (Sox9) and counterstained with hematoxylin. In contrast, RUNX2 knock-down (right) pellets regularly failed to form good cartilage differentiation (Alcian blue/Hematoxylin). (d) Western Blot analysis of C3 MSCs infected with control lentivirus or shRUNX2 lentivirus. RUNX2 protein is effectively knocked-down. (B) RUNX2 knock-down in human C3 MSCs supporting hematopoietic cells and progenitors in LTCs. Cord blood CD34+ cells were plated onto C3-MSC layers. Weekly cultures were halved to be reFed, harvested cells were counted (shown in a) and replated in semisolid hematopoietic secondary cultures to evaluate their progenitor cell content (b). Graphs derive from one of three different experiments performed in triplicates. C3 MSCs represent cells infected with missense control viral particles. BM6-MSCs are included as standard hematopoietic supportive cells. The treatment did not affect the overall survival of hematopoietic cells (a), but selectively decreased the hematopoietic progenitors output (b). At wk 5 the culture was terminated harvesting both the cells in suspension (s) and the ones entrapped in the adherent layer (a), which were cumulated to generate the points wk5 $(\mathrm{s}+\mathrm{a})$. 
emerging as a biological rather than a conceptual entity, osteoblasts, and reticular stromal cells, all belonging to the MSCs family have been shown, in vivo, as the only candidates defining this compartment [29,26,31,35,25]. The closest anatomic counterparts to the concept of "hematopoietic niche" are the blood islands around the developing embryo or the erythroblast islands in the red BM.

Thus, the effect of miR-204\&211 on RUNX2, and the consequent inhibition of osteogenesis, could impact the whole MSC differentiation spectrum, including cartilage and hematopoietic support. C3 MSCs are a colonic trilineage precursor primary line derived from $\mathrm{SMA}^{+}$pericryptalmyofibroblasts [30] able to differentiate in osteoblasts, chondroblasts and reticular stromal cells supporting hematopoiesis, but not toward adipocytes. Their baseline expression of miR-204\&211 is very low, similar to BM-MSCs, which matches with their stemness functional similarities. On the other hand, C6 MSCs represent a colonic uni-lineage precursorprimary line derived from SMA- inter-cryptal myofibroblasts [30,24,2], with very restricted differentiation potential. Accordingly, their baseline expression of miR-204\&211 is high. It is thus conceivable that miR-204\&211 expression could regulate stem cell progression and lineage restriction. C3 MSCs were chosen as an adequate cell model to confirm (as in [14] for osteo/adipo switch) and extend (present report for chondro/hematopoietic support inhibition) the effect of miR-204\&211 over-expression in human MSCs.

Adipogenesis, absent in C3 MSCs, is restored, at least in part, by miR 204 or 211 gene transfer, which in turn, impairs not only osteogenesis, but also chondrogenesis and hematopietic supportive activity in 5wk LTCs. C3-MSCs, transduced with miR 204 and miR 211, strongly downmodulate RUNX2 protein, but not CRTAP, a cartilage specific potential targetfor miR- 204/211 (www.targets can.org). As a single gene product can be targeted by multiple miRNA species, and a single microRNA can target multiple mRNAs [7,28,32,1,22,34,13,3,15,38,9], additional targets of miR-204\&211 might account for the disruption of chondrogenesis and hematopoietic supporting activity. Experiments with stable RUNX2 shRNA were performed to rule out the role for other targets of miR-204\&211 impaired chondrogenesis and hematopoietic supporting activity. These permanent knock-down experiments fully recapitulated the phenotypes observed with miR-204\&211, thus demonstrating that RUNX2 itself is necessary for normal chondrogenesis and to support hematopoiesis in vitro. It would be of great interest to validate the role of RUNX2 in hematopoietic supportive activity, as well as for the entire MSC differentiation spectrum in an in vivo system $[26,31,25]$.

The hematopoietic supporting activity exerted by an intestinal MSCs subtype is puzzling. It is possible that this function, originally described in BM-MSCs, could have adapted in peri-cryptal intestinal myofibroblasts to nurture intestinal stem cells within the crypts (intestinal niches), but is versatile enough to support also hematopoietic cells. The recent discovery of platelet generation and ectopic hematopoiesis in lung [16] corroborates this hypothesis. Furthermore, stress hematopoiesis, as known, can take place in ectopic sites in Cooley's anemia leading to facial deformity. MSC supporting activity in tissue specific niches for highly demanded lineages, like the hematopoietic and the intestinal epithelia, might allow permissive cross-lineage colonization, as indicated by their functional deployment by adenocarcinoma cells [19], or BM diffusing neuroblastomas. We could thus speculate that some circulating white hematopoietic precursor might, when necessary, utilize peri-cryptal myofibroblasts, as nurturing pseudo-crypt for a local quick expansion [21]. This fact highlights the importance of the MSC-determined microenvironment in maintainingnormal homeostasis as well as inparticipating in inflammatory diseases (Crohn's disease) and in cancer progression.

Our data are not in contrast with a former report [10] showing no major chondrogenic impair by transient RUNX2 knock-down in a murine cell line. Those data, obtained with a different technique, might have knockeddown RUNX2 too shortly to produce the chondrogenic inhibition. It is thus apparent that RUNX2, a recognized master gene for osteogenesis, sits at the intersection of multiple MSC pathways regulating commitment and differentiation toward all canonical MSC fates. MiR$204 \& 211$ have been already involved in osteoporosis [14] and obesity $[23,6]$. Our additional data, i.e., inhibition of chondrogenesis and hematopoietic supporting activity, coupled with promotion of adipogenesis and depression of osteogenesis, strikingly recall the clinical traits of metabolic syndrome, where obesity, osteoarthritis, osteoporosis and anaemia are all combined. This suggests that miR-204\&211-RUNX2 axis in human MSCs might play a significant role in the pathogenesis of this rapidly increasing disease in technologically advanced areas, and indicate a possible pharmacologic approach to regenerate homeostasis.

Acknowledgements. We are grateful to Agnese D'Angiò for isolating hematopoietic progenitor cells from cord blood. None of the authors has any conflict of interest.

\section{References}

1. Ballas N, Grunseich C, Lu D, Speh J, Mandel G (2005), REST and its co-repressor mediate plasticity of neuronal gene chromatin throghout neurogenesis. Cell 121, 645-657.

2. Barbanti Brodano G, Terzi S, Trombi L, Griffoni C, Valtieri M, Boriani S, Magli MC (2013), Mesenchymal stem cells derived from vertebrae (vMSCs) show best biological properties. Eur Spine J 22, S979-84.

3. Bartel DP (2004), MicroRNAs: genomics, biogenesis, mechanism, and function. Cell 116, 281-297.

4. Bruderer M, Richards RG, Alini M, Stoddart MJ (2014), Role and regulation of RUNX2 in osteogenesis. Eur Cell Mater. 28, 269-86.

5. Cole AG (2011), A review of diversity in the evolution and development of cartilage: the search for the origin of the chondrocyte. Eur Cells Mater 21, 122-129. 
6. Civelek M, Hagopian R, Pan C, Che N, Yang WP, Kayne PS, Saleem NK, Cederberg H, Kuusisto J, Gargalovic PS, Kirchgessner TG, Laakso M, Lusis AJ (2013), Genetic regulation of human adipose microRNA expression and its consequences for metabolic traits. Hum Mol Genet 22, 3023-3037.

7. De Marchis ML, Ballarino M, Salvatori B, Puzzolo MC, Bozzoni I, Fatica A (2009), A new molecular network comprising PU.1, interferon regulatory factor proteins and miR-342 stimulate ATRA- mediated granulocytic differentiation of acute promyelocyticleukemia cells. Leukemia. 23, 856-862.

8. Gawlitta D, Farrell E, Malda J, Creemers LB, Alblas J, Wouter JA, Dhert WJA (2010), Modulating Endochondral Ossification of Multipotent Stromal Cells for Bone Regeneration. Tissue Eng: B 16, 385-95.

9. Gibson G, Asahara H (2013), MicroRNAs and cartilage. J Orthop Res 31, 1333-1344.

10. Gordeladze JO, Noel D, Bony C, Apparailly F, Louis-Plence $\mathrm{P}$, Jorgensen C (2008), Transient down-regulation of cbfa1 Runx2 by RNA interference in murine C3H10T1/2 mesenchymal stromal cells delays in vitro and in vivo osteogenesis, but does not overtly affect chondrogenesis. Exp Cell Res 314, 1495-506.

11. Gordeladze JO, Djouad F, Brondello JM, Noël D, DurouxRichard I, Apparailly F, Jorgensen C (2009), Concerted stimuli regulating osteo-chondral differentiation from stem cells: phenotype acquisition regulated by microRNAs. ActaPharmacol Sin 30, 1369-1384.

12. Gorleladze JO, Reseland JE, Karlsen TA, Jakobsen RB, Stunes AK, Syversen U, Engebretsen L, Lyngstadaas SP, Jorgensen C (2013), Engineering of inflammation-resistant osteochondral cells, in: JA. Andrades (ed), Regenerative medicine and tissue engineering. InTech, ISBN 978-953-511108-5.

13. Gregory RI, Chendrimada TP, Shiekhattar R. (2006), MicroRNA biogenesis: isolation and characterization of the microprocessor complex. Methods mol biol 342, 33-47.

14. Huang J, Zhao L, Xing L, Chen D (2010), MicroRNA-204 Regulates Runx2 Protein Expression and Mesenchymal Progenitor Cell Differentiation. Stem Cells 28, 357-364.

15. Kobayashi T, Lu J, Cobb BS, Rodda SJ, McMahon AP, Schipani E, Merkenschlager M, Kronenberg HM (2008), Dicer-dependent pathways regulate chondrocyte proliferation and differentiation. Proc Natl Acad Sci U S A 12, 1949-1954.

16. Lefrançais E, Ortiz-Muñoz G, Caudrillier A, Mallavia B, Liu F, Sayah DM, Thornton EE, Headley MB, David T, Coughlin SR, Krummel MF, Leavitt AD, Passegué E, Looney MR (2017), The lung is a site of platelet biogenesis and a reservoir for haematopoietic progenitors. Nature doi:10.1038/nature21706

17. Lian JB, Stein GS, van Wijnen AJ, Stein JL, Hassan MQ, Gaur T, Zhang Y (2012), MicroRNA control of bone formation and homeostasis. Nat Rev Endocrinol 8, 212-227.

18. Lin L, Shen Q, Leng H, Duan X, Fu X, Yu C (2011), Synergistic Inhibition of Endochondral Bone Formation by Silencing Hif1 $\alpha$ and Runx2in Trauma-induced Heterotopic Ossification. Mol Ther 19, 1426-32.

19. Lugini L, Valtieri M, Federici C, Cecchetti S, Meschini S, Condello M, Signore M, Fais S (2016), Exosomes from human colorectal cancer induce a tumor-like behavior in colonic mesenchymal stromal cells. Oncotarget. 7, 50086-50098.

20. Mangiavacchi A, Sorci M, Masciarelli S, Larivera S, Legnini I, Iosue I, Bozzoni I, Fazi F, Fatica A (2016), The miR-223 host non-coding transcript linc-223 induces IRF4 expression in acute myeloid leukemia by acting as a competing endogenous RNA. Oncotarget 7, 60155-60168.
21. Messina V, Buccione C, Marotta G, Ziccheddu G, Signore M, Mattia G, Puglisi R, Sacchetti B, Biancone L, Valtieri M (2017), Gut mesenchymal stromal cells in immunity. Stem Cell Int. Article ID 8482326

22. Ng F, Boucher S, Koh S, Sastry SR, Chase L, Lakshmipathy U, Choong C, Yang Z, Vemuri MC, Rao MS, Tanavde V (2008), PDGF, TGF-b and FGF signaling is important for differentiation and growth of mesenchymal stem cells (MSCs): transcriptional profiling can identify markers and signaling pathways important in differentiation of MSC into adipogenic, chondrogenic and osteogenic lineages. Blood 112, 295-307.

23. O'Neill S, Bohl M, Gregersen S, Hermansen K, O'Driscoll L (2016), Blood-Based Biomarkers for Metabolic Syndrome. Trends Endocrinol Metab 27, 363-374.

24. Picchi J, Trombi L, Spugnesi L, Baracchini S, Maroni G, Barbanti Brodano G, Boriani S, Valtieri M, Petrini M, Magli MC (2013), HOX and TALE Signatures Specify Human Stromal Stem Cell Populations From Different Sources. J Cell Physiol 228, 879-889.

25. Reinish A, Thomas D, Corces MR, Zhang X, Gratzinger D, Shallmoser W-JK, Strunk D, Majeti R (2016), A humanized bone marrow ossiclexeno transplant model enables improved engraftment of healhy and leukemic human hematopoietic cells. Nat Med 22, 812-821.

26. Sacchetti B, Funari A, Michienzi S, Di Cesare S, Piersanti S, Saggio I, Tagliafico E, Ferrari S, Robey PG, Riminucci M, Bianco P (2007), Self-renewing osteoprogenitors in bone marrow sinusoids can organize a hematopoietic microenvironment. Cell 131, 324-336.

27. Sacchetti B, Funari A, Remoli C, Giannicola G, Kogler G, Liedtke S, Cossu G, Serafini M, Sampaolesi M, Tagliafico E, Tenedini E, Saggio I, Robey PG, Riminucci M, Bianco P (2016), No identical "mesenchymal stem cells" at different times and sites: committed progenitors of distinct origin and differentiation potential are incorporated as adventitial cells in microvessels. Stem Cell Rep 14 6, 897-913.

28. Salvatori B, Iosue I, Mangiavacchi A, Loddo G, Padula F, Chiaretti S, Peragine N, Bozzoni I, Fazi F, Fatica A (2012), The microRNA-26a target E2F7 sustains cell proliferation and inhibits monocytic differentiation of acute myeloid leukemia cells. Cell Death Dis 3, e413.

29. Scadden DT (2014), Nice neighborhood: emerging concepts of the stem cell niche. Cell 157, 41-50.

30. Signore M, Cerio AM, Boe A, Pagliuca A, Zaottini V, Schiavoni I, Fedele G, Petti S, Navarra S, Ausiello MC, Pelosi E, Fatica A, Sorrentino A, Valtieri M (2012), Identity and ranking of colonic mesenchymal stromal cells. J Cell Physiol 227, 3291-3300

31. Serafini M, Sacchetti B, Pievani A, Redaelli D, Remoli C, Biondi A, Riminucci M, Bianco P (2014), Establishment of bone marrow and hematopoietic niches in vivo by reversion of chondrocyte differentiation of human bone marrow stromal cells. Stem Cell Res 12, 659-672.

32. Singh SK, Kagalwala MN, Parker-Thornburg J, Adams H, Majumder S (2008), REST/NRSF maintains self-renewal and pluripotency of embryonic stem cells. Nature 453, 223-227.

33. Sorrentino A, Ferracin M, Castelli G, Biffoni M, Tomaselli G, Baiocchi M, Fatica A, Negrini M, Peschle C, Valtieri M (2008), Isolation and characterization of CD146+ multipotent mesenchymal stromal cells. Exp Hematol 36, 1035-1046.

34. Stadler BM, Ruohola-Baker H (2008), Small RNAs: keeping stem cells in line. Cell 132, 563-566.

35. Tamplin OJ, Durand EM, Carr LA, Childs SJ, Hagedorn EJ, Li P, Yzaguirre AD, Speck NA, Zon LI (2015), Hematopoietic stem cell arrival triggers dynamic remodeling of the perivascular niche. Cell 160, 241-252. 
36. Valtieri M, Schiro' R, Chelucci C, Masella B, Testa U, Casella I, Montesoro E, Mariani G, Hassan HJ, Peschle C (1994), Efficient transfer of selectable and membrane reporter genes in hematopoietic progenitor and stem cells purified from human peripheral blood. Cancer Res 54, 4398-4404.

37. Valtieri M, Sorrentino A (2008), The Mesenchymal Stromal Cell Contribution to Homeostasis. J Cell Physiol. 217, 296-300.
38. Zhang R, Ma J, Yao J (2013), Molecular mechanisms of the cartilage-specific microRNA-140 in osteoarthritis. Inflamm Res 62, 871-877.

39. Ziegler BL, Valtieri M, AlmeidaPorada G, De Maria R, Müller R, Masella B, Gabbianelli M, Casella I, Pelosi E, Bock T, Zanjani ED, Peschle C (1999), KDR receptor: a key marker defining hematopoietic stem cells. Science 285, 1553-8.

Cite this article as: Sacchetti B, Fatica A, Sorci M, Sorrentino A, Signore M, Cerio A, Felicetti F, Feo AD, Pelosi E, Caré A, Pescarmona E, Gordeladze JO, \& Valtieri M (2017) Effect of miR-204\&211 and RUNX2 control on the fate of human mesenchymal stromal cells. Regen Med Res, 5, 2 